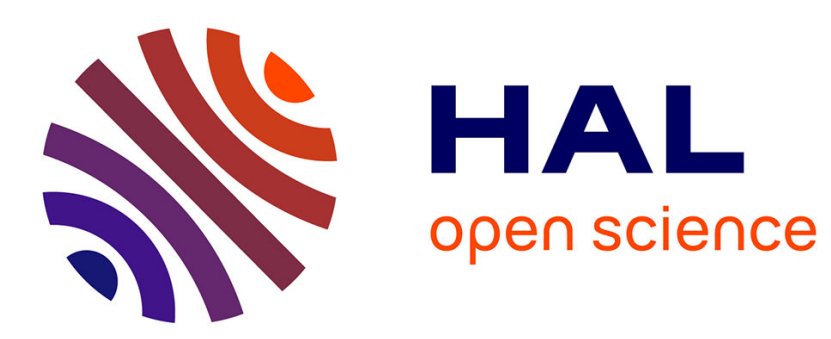

\title{
Dispositif de mesures du pouvoir thermoélectrique sur des échantillons très résistants entre 4 et $300 \mathrm{~K}$
}

\author{
Patrice Dordor, Emile Marquestaut, Gérard Villeneuve
}

\section{To cite this version:}

Patrice Dordor, Emile Marquestaut, Gérard Villeneuve. Dispositif de mesures du pouvoir thermoélectrique sur des échantillons très résistants entre 4 et $300 \mathrm{~K}$. Revue de Physique Appliquée, 1980, 15 (11), pp.1607-1612. 10.1051/rphysap:0198000150110160700 . jpa-00244888

\section{HAL Id: jpa-00244888 https://hal.science/jpa-00244888}

Submitted on 1 Jan 1980

HAL is a multi-disciplinary open access archive for the deposit and dissemination of scientific research documents, whether they are published or not. The documents may come from teaching and research institutions in France or abroad, or from public or private research centers.
L'archive ouverte pluridisciplinaire HAL, est destinée au dépôt et à la diffusion de documents scientifiques de niveau recherche, publiés ou non, émanant des établissements d'enseignement et de recherche français ou étrangers, des laboratoires publics ou privés. 


\title{
Dispositif de mesures du pouvoir thermoélectrique sur des échantillons très résistants entre 4 et $300 \mathrm{~K}$
}

\author{
P. Dordor, E. Marquestaut et G. Villeneuve \\ Laboratoire de Chimie du Solide du CNRS, Université de BordealuI I. \\ 351, cours de la Libération, 33405 Talence Cedex, France.
}

(Reçu le 19 mai 1980, révisé le 21 juillet 1980, accepté le $1^{\mathrm{er}}$ août 1980)

\begin{abstract}
Résumé. - L'appareil décrit dans le texte est conçu pour mesurer le pouvoir thermoélectrique de monocristaux de forte résistivité $\left(\simeq 10^{10} \Omega \mathrm{cm}\right.$ ) entre 4 et $300 \mathrm{~K}$. Il permet d'effectuer des mesures sur des échantillons de faibles dimensions $(<0,5 \mathrm{~mm})$. Le dispositif a été testé sur un monocristal de magnétite de composition stœchiométrique. Bien que non construit pour, il est capable de mesurer le coefficient de Seebeck d'échantillons métalliques pour lesquels $\alpha<10 \mu \mathrm{V} /{ }^{\circ} \mathrm{C}$.
\end{abstract}

Abstract. - A new method is described for dynamically measuring the thermoelectric power of small samples in the temperature range 4-300 K. Measurements may be performed on samples with resistivities as high as $10^{10} \Omega \mathrm{cm}$. The device has been checked on a crystal of stoichiometric magnetite. Although designed for highly resistive materials, it may be used for Seebeck measurements on metallic samples with $\alpha<10 \mu \mathrm{V} /{ }^{\circ} \mathrm{C}$.

1. Introduction. - La mesure de la variation du pouvoir thermoélectrique (coefficient de Seebeck) en fonction de la température, couplée avec celle de la conductivité électrique, permet souvent de caractériser les mécanismes de conduction dans les solides [1].

Cependant les appareillages classiques ne permettent pas d'effectuer des mesures correctes dès que la conductivité de l'échantillon devient inférieure à $10^{-5} \Omega^{-1} \mathrm{~cm}^{-1}$, ce qui restreint les possibilités d'exploitation, surtout pour les semi-conducteurs à basse température. En outre, il est toujours plus fécond d'opérer sur des monocristaux, et sauf cas particuliers, ceux-ci ont le plus souvent de faibles dimensions $\left(<1 \mathrm{~mm}^{3}\right)$, ce qui apporte une difficulté supplémentaire à la mesure.

C'est ainsi que nous avons conçu et réalisé un appareillage permettant de mesurer le pouvoir thermoélectrique d'échantillons très résistants $(\sigma$ pouvant atteindre $10^{-9}$ ou $10^{-10} \Omega^{-1} \mathrm{~cm}^{-1}$ ) sur des cristaux dont les dimensions peuvent être inférieures à $0,5 \mathrm{~mm}$. Son domaine d'application s'étend pour le moment de 4,2 à $300 \mathrm{~K}$.

La détermination du coefficient de Seebeck $\alpha$ implique une mesure du gradient thermique $\Delta T$ existant entre les extrémités de l'échantillon, et de la force électromotrice $\Delta V$ correspondante. Chacune de ces deux mesures ne doit évidemment pas perturber l'autre.

La mesure de $\Delta V$ doit être effectuée en circuit ouvert.
Cette dernière condition impose que le système de mesures ait une résistance équivalente très supérieure à celle des échantillons étudiés.

Ces impératifs conditionnent la conception de l'appareillage :

(i) L'élimination des potentiels de contact impose une mesure de $\Delta T$ variable (inversion ou modulation). Pour des raisons de sensibilité nous avons choisi la modulation (mesure dynamique). Dans notre cas, cette méthode n'était pas concevable à partir de microfours à résistance chauffante. En effet, lorsque la résistance des échantillons dépasse $10^{7} \Omega$, les variations de courant dans l'enroulement créent, par rayonnement dans le circuit de mesures de $\Delta V$, des tensions parasites non négligeables. Nous avons préféré, pour créer le gradient thermique, une méthode optique focalisant sur la zone chaude un faisceau lumineux, solution déjà proposée par Freeman et Bass [2], puis par Keem [3]. Nous avons introduit en outre une modulation du flux, dont les avantages sont décrits au chapitre 2 .

(ii) Le fait que la mesure de $\Delta V$ doit être effectuée en circuit ouvert, impose au circuit de mesures une résistance équivalente théoriquement infinie, et en pratique supérieure de plusieurs ordres de grandeur à celle des échantillons.

Dans la deuxième partie de ce mémoire, nous 
décrivons la cellule de mesures construite à partir de ces considérations. La troisième partie du texte est consacrée à la description des circuits électroniques permettant de mesurer $\Delta V$ et $\Delta T$. Enfin l'appareillage est testé sur un échantillon de magnétite $\mathrm{Fe}_{3} \mathrm{O}_{4}$, sur lequel des mesures ont déjà été effectuées à l'aide d'un dispositif basé sur un principe totalement différent.

2. Description de la cellule. - Le schéma de la cellule est représenté à la figure 1 .

L'échantillon à tester est maintenu par pincement entre un cylindre de cuivre (a), qui constitue la source froide, et un bloc de carbone (b) chemisé de cuivre, jouant le rôle de source chaude.

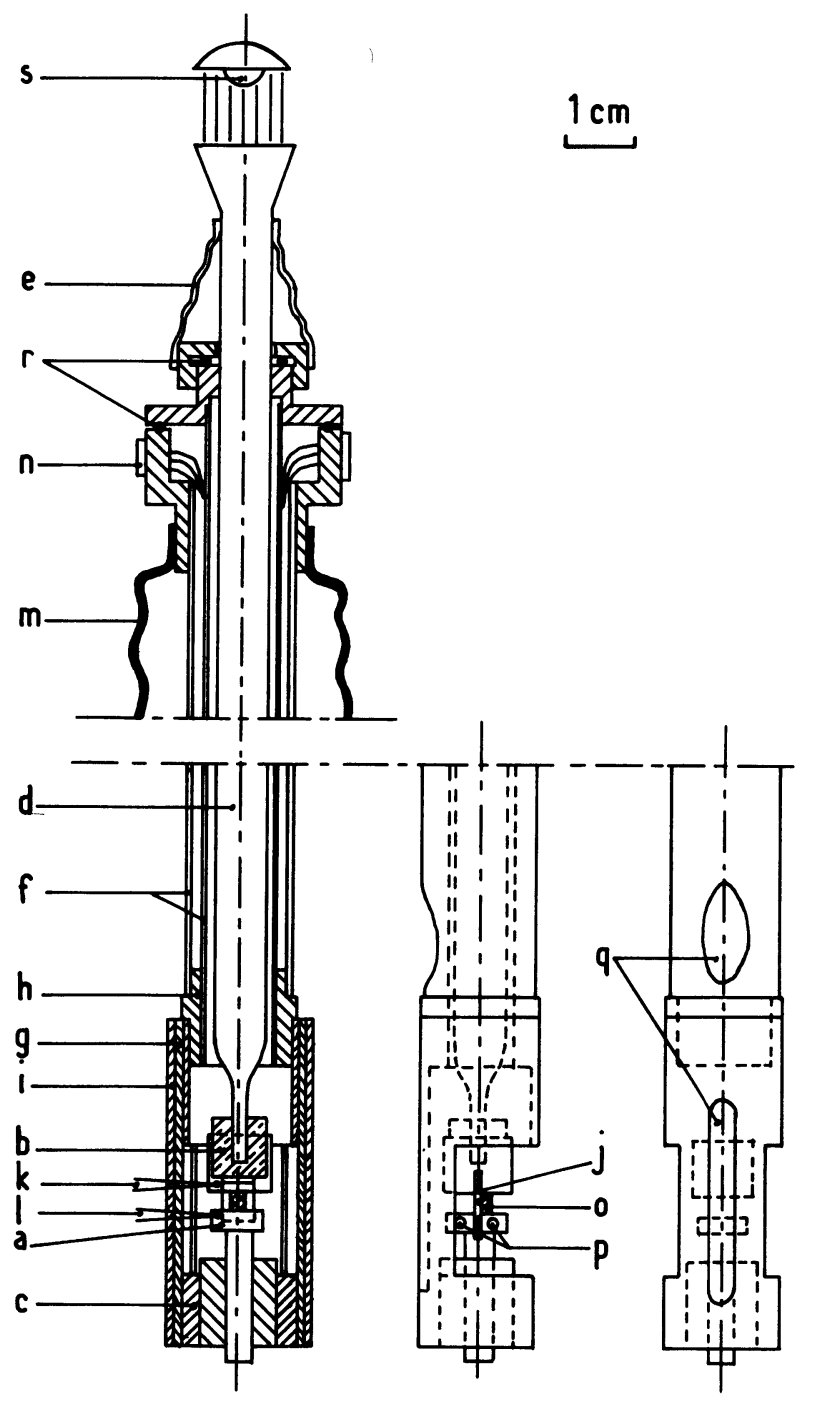

Fig. 1. - Schéma de la cellule. a : point froid, $b$ : bloc de carbone, chemisé de cuivre, $\mathrm{c}$ : corps de la cellule (laiton), $\mathrm{d}$ : tige de quartz, $\mathrm{e}:$ ressorts, $\mathrm{f}$ : tube en acier inox, $\mathrm{g}$ : écran en cuivre électrolytique, $\mathrm{h}:$ isolateur (téflon), $\mathrm{i}:$ gaine (téflon), $\mathrm{j}:$ support (saphir), $\mathrm{k}, 1$ : thermocouples Au-Fe, chromel, $\mathrm{m}$ : soufflet d'étanchéité, $\mathrm{n}$ : connecteur BNC, $\mathrm{o}$ : échantillon, $\mathrm{p}$ : capteur de température, $q$ : lumière, $r$ : joint torique, $s:$ source lumineuse.

[Cell assembly. a : cold point, b : copper coated carbon block, $\mathrm{c}$ : brass holder, $\mathrm{d}:$ quartz rod, $\mathrm{e}:$ spring, $\mathrm{f}$ : inox. steel tubing, $g$ : OFHC copper shield, $h$ : teflon isolator, $i:$ teflon sheath, $j$ : sapphire holder, k, $1: \mathrm{KP}$ vs. Au-Fe thermocouple, $\mathrm{m}:$ tightness bellows, $\mathrm{n}:$ BNC connector, $\mathrm{o}:$ sample, $\mathrm{p}:$ temperature sensor, $\mathrm{q}:$ slot, $\mathrm{r}:$ : $\mathrm{o} »$ ring, $\mathrm{s}$ : light source.]
Le cylindre de cuivre est fixé sur le corps en laiton de la cellule (c). Le bloc de carbone est collé sur l'extrémité d'une longue tige de quartz (d). Celle-ci sert de guide de lumière à un rayonnement infra-rouge focalisé à son extrémité supérieure. Transmis avec une faible atténuation par le quartz, le rayonnement lumineux est suffisamment intense pour élever la température du bloc de carbone de plusieurs degrés. La tige de quartz, mises à part ses deux extrémités, est métallisée de manière à éviter que l'échantillon ne reçoive une fraction du rayonnement lumineux réémis par le quartz. Des ressorts (e) fixés sur la partie supérieure de la cellule, exercent sur la tige de quartz une pression axiale, qui maintient l'échantillon plaqué contre la source froide. Ce système de blocage présente l'avantage d'être insensible aux dilatations de la cellule sous l'effet de la température.

Deux tubes concentriques (f), en acier inoxydable, soutiennent le corps de la cellule. Pour assurer un isolement électrique entre la cellule et son support, la liaison entre ces deux parties est réalisée en téflon.

La cellule est introduite dans un réservoir à hélium liquide. La distance entre la cellule et le bain détermine la température moyenne de l'échantillon. Ce procédé de régulation permet d'obtenir rapidement un équilibre thermique suffisant : au bout de $15 \mathrm{~min}$., les fluctuations de température sont inférieures à $0,3 \mathrm{~K}$ pendant la durée de la manipulation; un tel résultat suppose bien entendu que l'évacuation de l'hélium liquide se fasse très régulièrement. Deux gaines concentriques montées sur le corps de la cellule, contribuent à cette stabilité : la première, métallique (g) augmente l'inertie thermique, la seconde en téflon (i), atténue l'effet des courants de convection.

Une plaquette de saphir $(\mathrm{j})$, intercalée entre les blocs (a) et (b), permet un positionnement aisé des échantillons, même quand ils sont de petites dimensions. La résistivité élevée $\left(10^{16} \Omega \mathrm{cm}\right)$ du saphir assure un excellent isolement entre les deux blocs. La résistance d'isolement de la cellule ainsi réalisée est supérieure à $10^{13} \Omega$. La fuite thermique par l'intermédiaire de cette plaquette contribue à diminuer le gradient thermique qui s'établit spontanément entre les extrémités chaude et froide de l'échantillon. Dans ces conditions, la valeur du gradient reste inférieure à $0,5 \mathrm{~K}$ pour des échantillons de longueur inférieure à $1 \mathrm{~mm}$.

Cependant, lorsque la longueur de l'échantillon dépasse plusieurs millimètres, un gradient thermique de quelques degrés s'établit naturellement, il reste approximativement constant dans le domaine de température exploré. Une résistance de carbone $(50 \Omega$, $1 / 16 \mathrm{~W}$ ), noyée dans le bloc froid et parcourue par un courant constant, permet d'annuler ce gradient.

Les fils de liaisons, nécessaires à la mesure de la tension $\Delta V$, sont soudés sur les deux blocs chaud et froid, à l'aide d'un alliage (CEROBEN) comportant un faible coefficient de Seebeck, de façon à ne pas ajouter de tension parasite dans la mesure. 
Malgré les précautions précédentes on mesure, en l'absence de gradient thermique, des forces électromotrices de contact de l'ordre de quelques microvolts. La détermination du pouvoir thermoélectrique, par une seule mesure du gradient thermique et du potentiel associé, conduit donc à une erreur systématique.

Pour s'affranchir de cette erreur nous avons choisi une méthode de mesures dynamique : nous imposons une vitesse de chauffe et nous mesurons, en fonction du temps, la variation de tension $\Delta V$.

Si l'on veut contrôler le taux de croissance de l'excitation lumineuse, il ne faut pas commander la lampe en tout ou rien, mais en moduler le flux. Un générateur de signaux de très basse fréquence, fonctionnant en monocoup et suivi d'un amplificateur de puissance, permet de modifier progressivement l'énergie rayonnée.

La mesure des échauffements des deux blocs chaud et froid, est réalisée à l'aide de thermocouples or fer-chromel $(\varnothing=0,01 \mathrm{~mm})$. Un ancrage thermique important permet de minimiser la fuite thermique par les fils de thermocouples; cette opération nécessaire en raison de la faible masse de l'ensemble bloc chaudéchantillon $(m<1 \mathrm{~g})$. Les thermocouples sont noyés dans les sources chaude et froide à moins de $0,2 \mathrm{~mm}$ de l'échantillon.

La mesure de la température absolue de l'échantillon est effectuée à l'aide de deux résistances, l'une de platine et l'autre de carbone. Celles-ci sont incluses dans le bloc froid, à proximité immédiate de l'échantillon.

3. Electronique de mesure. - 3.1 Conditions INHÉRENTES AUX MESURES EN CIRCUIT OUVERT. Soumis à un gradient thermique, un échantillon est équivalent à un générateur possédant une force électromotrice $V_{\mathrm{s}}$ et une résistance $R$. Pour mesurer la tension $V_{\mathrm{s}}$ sans atténuation, il est nécessaire que l'ensemble de l'équipement électronique associé à la cellule possède une impédance d'entrée $Z_{\mathrm{i}} \gg R$ (si $R \simeq 10^{10} \Omega, Z_{\mathrm{i}} \gg 10^{12} \Omega$ ).

L'amplitude du bruit aux bornes d'un échantillon de résistance $R$, relié aux entrées d'un étage amplificateur, est donnée par la relation ci-après, dans laquelle on suppose que les sources de bruit sont non corrélées :

$$
\bar{e}_{\mathrm{n}}=\sqrt{\bar{E}_{\mathrm{n}}^{2}+R^{2} \bar{I}_{\mathrm{n}}^{2}+4 k T B R},
$$

avec

$\bar{e}_{\mathrm{n}}$ : valeur efficace de la tension de bruit à l'entrée de l'amplificateur,

$\bar{E}_{\mathrm{n}}$ : tension de bruit propre de l'amplificateur,

$\bar{I}_{\mathrm{n}}$ : courant de bruit de l'amplificateur,

$k$ : constante de Boltzmann,

$B$ : bande passante de l'appareil de mesures.

Lorsque la résistivité augmente, le terme $R^{2} \bar{I}_{\mathrm{n}}^{2}$ devient vite prépondérant, accroissant considérablement le bruit ramené à l'entrée de l'amplificateur. Seuls les électromètres comportent jusqu'ici des cou- rants de bruit $\bar{I}_{\mathrm{n}}$ et de polarisation $\bar{I}_{\mathrm{p}}$ très inférieurs au picoampère.

Pour les amplificateurs paramétriques à varactor utilisés (3431 BURR BROWN) ces grandeurs valent respectivement :

$$
I_{\mathrm{p}}: 10^{-15} \mathrm{~A}, \bar{I}_{\mathrm{n}} \leqslant 10^{-15} \mathrm{~A} \text { p.p., } \bar{E}_{\mathrm{n}} \leqslant 5 \mu \mathrm{V} \text { p.p., }
$$
pour des fréquences de fonctionnement $<10 \mathrm{~Hz}$.

La tension de bruit en est inférieure à $5 \mu \mathrm{V}$ si

$$
R=10^{10} \Omega, \quad T=300 \mathrm{~K} \quad \text { et } B=1 \mathrm{~Hz} .
$$

-

Dans les conditions de l'exemple précédent, la chute ohmique dans l'échantillon $R I_{\mathrm{p}}$ est donc de l'ordre de $100 \mu \mathrm{V}$, valeur qui n'est nullement négligeable pour de faibles valeurs du coefficient de Seebeck.

\subsection{ELECTRONIQUE ASSOCIÉE A LA MESURE DE $\Delta V$. -} L'amplificateur intervenant dans la mesure de $\Delta V$ (Fig. 2) se compose de quatre parties principales.

A - L'étage d'entrée, réalisé à l'aide d'un électromètre, possède une structure d'amplificateur non inverseur. Cette configuration lui assure une grande impédance d'entrée (pour $f<10^{-1} \mathrm{~Hz}, Z_{\mathrm{i}}>10^{13} \Omega$ ). Nous n'avons pas choisi de montage différentiel, car celui-ci perd de son intérêt du fait de la différence de résistance des sources. Le gain se limite à un ordre de grandeur de 10 si on désire une stabilité convenable. Compte tenu de la constante de temps thermique de la cellule et de la capacité parasite des fils de jonction, la fréquence de coupure à $-3 \mathrm{~dB}$ est fixée à $1 \mathrm{~Hz}$. Le point froid de l'échantillon, pris comme zéro électrique, est directement relié à la masse du préamplificateur.

La capacité parasite de la cellule et des fils de liaison $(200 \mathrm{pF})$ limite sa réponse en fréquence. Le temps d'établissement de la tension de Seebeck est fixé, en première approximation, par la constante de temps $\tau=R C_{\mathrm{p}}$, produit de la résistance de l'échantillon par la capacité parasite de la cellule. Dans un circuit du premier ordre, le signal s'établit à $99 \%$, au bout d'un temps égal à $5 \tau$. Si l'on souhaite que la tension $\Delta V$ atteigne une valeur d'équilibre aux bornes d'un échantillon de résistance $R=10^{10} \Omega$, il faut maintenir le gradient thermique pendant au moins $10 \mathrm{~s}$

B - Pour réduire l'influence de la capacité parasite, nous utilisons la technique de la garde active : un potentiel image de la tension d'entrée est disponible

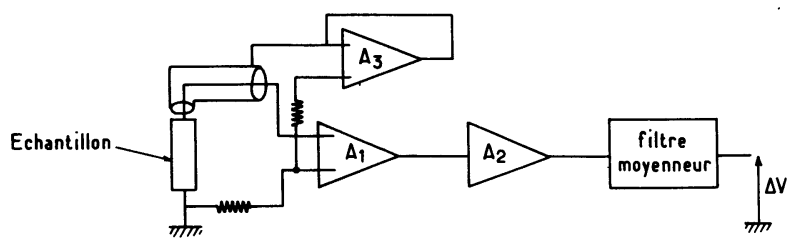

Fig. 2. - Mesure de la tension de Seebeck : schéma de l'amplificateur.

[Schematic representation of Seebeck voltage measurement]. 
sous basse impédance, sur l'entrée inverseuse de l'amplificateur d'entrée. L'amplificateur $A_{3}$ (Fig. 2), monté en suiveur de tension, pilote la garde des câbles reliant la cellule aux circuits électroniques traitant les différentes informations provenant du bloc chaud. La tête de la cellule et les tubes d'acier inoxydable sont portés à ce même potentiel.

$\mathrm{C}-$ Le second étage amplificateur $\mathrm{A}_{2}$, de type inverseur, donne à la voie de mesure de la tension de Seebeck un gain de 100.

D - L'amplitude du bruit, à la sortie de ce circuit, est supérieure à la valeur précédemment calculée. $\mathrm{La}$ relation utilisée ne tenait compte en effet ni des vibrations de la cellule, entraînant la fluctuation des capacités parasites, ni du bruit provenant du contact échantillon-support.

Pour augmenter le rapport signal/bruit à la sortie de l'amplificateur $A_{2}$, nous utilisons les filtres décrits à la figure 3. Ce type de circuit proposé par Balslew [4] permet d'obtenir une valeur moyenne continue à chaque moment de la mesure. Pour optimiser la réponse de l'ensemble cellule-circuit électronique, il faut que les filtres aient une constante de temps du même ordre de grandeur que celle du circuit électrique équivalent à la cellule.

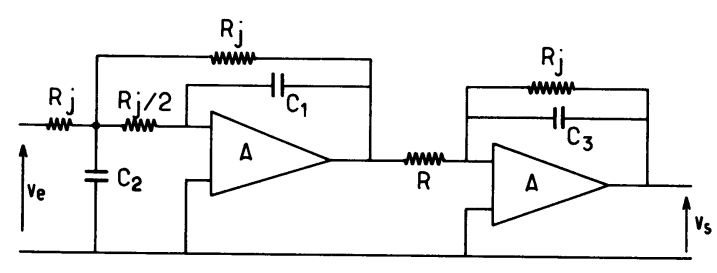

Fig. 3. - Filtre moyenneur.

[Continuous averaging filter.]

3.3 EleCtronique ASSOCIÉE A LA MESURE DE $\Delta T$. Les deux thermocouples (k) et (l), intervenant dans la mesure du gradient thermique $\Delta T$, sont noyés dans les blocs chaud et froid. Il n'est donc pas possible de les réunir directement en un montage différentiel, on court-circuiterait en effet l'échantillon.

Le rôle du circuit décrit à la figure 4 est de fournir une tension proportionnelle au gradient thermique. Ce circuit, de structure symétrique, se compose de deux amplificateurs d'instrumentation $\mathrm{A}_{4}$ et $\mathrm{A}_{4}^{\prime}$ reliés aux thermocouples (k) et (l). Les étages $A_{5}$ et $A_{5}^{\prime}$ sont de même nature que les étages d'entrée. L'amplificateur $A_{6}$ effectue la soustraction entre les signaux délivrés par les amplificateurs $A_{5}$ et $A_{5}^{\prime}$.

Dans la mesure de la température du bloc chaud, la tension de Seebeck intervient comme une tension de mode commun. Celle-ci pouvant être très supérieure à la f.é.m. délivrée par le thermocouple (k), l'amplificateur $\mathrm{A}_{4} \mathrm{~A}_{5} \mathrm{~A}_{6}$ doit avoir un fort taux de réjection de mode commun (T.R.M.C.).

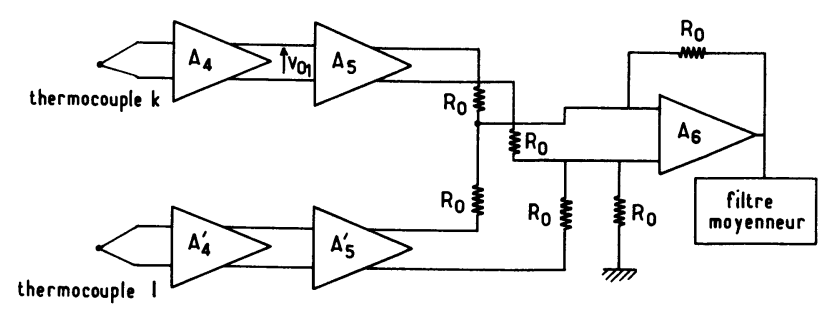

Fig. 4. - Mesure du gradient thermique, schéma de l'amplificateur.

[Block diagram of equipments for measuring thermal gradient.]

L'étage d'entrée $\mathrm{A}_{4}$, représenté à la figure 5 est réalisé à l'aide de deux électromètres. Le T.R.M.C. de cet étage amplificateur est supérieur à $100 \mathrm{~dB}$.

Le T.R.M.C. des étages $A_{5}$ et $A_{6}$ est déterminé par la relation :

$$
\text { T.R.M.C. }=\frac{\text { gain } \mathrm{A}_{5}}{4 k}\left(1+\text { gain } \mathrm{A}_{6}\right)
$$

où $k$ représente l'incertitude sur les résistances $R_{0}$ (Fig. 4). Pour une valeur donnée du gain total de l'ensemble $\left(A_{5}, A_{6}\right)$, le T.R.M.C. est d'autant plus élevé que le rapport $A_{5} / A_{6}$ est plus grand. Pour cette raison nous avons attribué aux étages $A_{4}, A_{5}, A_{6}$ les gains respectifs 10,100 et 1 . Dans ces conditions le T.R.M.C. global, mesuré pour une fréquence d'excitation de $0,1 \mathrm{~Hz}$, est supérieur à $80 \mathrm{~dB}$.

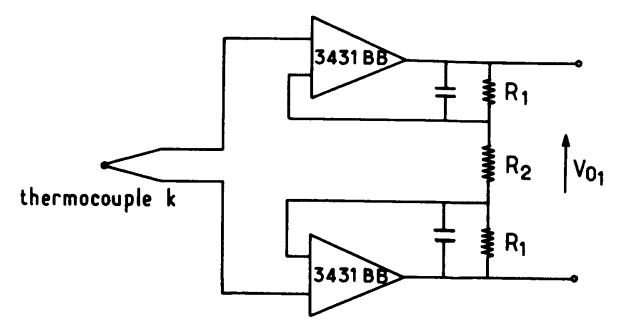

Fig. 5. - Mesure de la température du point chaud : schéma du circuit.

[Hot point temperature measurement : schematic circuit diagram.]

Soit $V_{\mathrm{t}}$ la variation de la tension associée à la fluctuation thermique du bloc chaud et $V_{\mathrm{s}}$ la tension entre la masse et le bloc chaud. L'erreur relative sur la mesure de $V_{\mathrm{t}}$, due au mode commun, est égale à $V_{s} / V_{t}$ T.R.M.C. Si le rapport $V_{s} / V_{t}$ est égal à 100 l'erreur de la tension, proportionnelle à la variation de température du bloc chaud, est inférieure à $1 / 100$.

Les fréquences de coupure des étages $\mathrm{A}_{4}$ et $\mathrm{A}_{4}^{\prime}$ doivent être identiques et égales d'ailleurs à celle de l'amplificateur $A_{1}$, qui mesure la tension de Seebeck.

Des filtres moyenneurs du même type que ceux précédemment décrits, équipent cette voie de mesure. L'appariement de ces deux séries de filtres est une condition nécessaire au bon fonctionnement du système de mesures.

3.4 Mise EN CEUVRE EXPÉRIMENTALE. - Une fois la température moyenne de l'échantillon stabilisée, il 


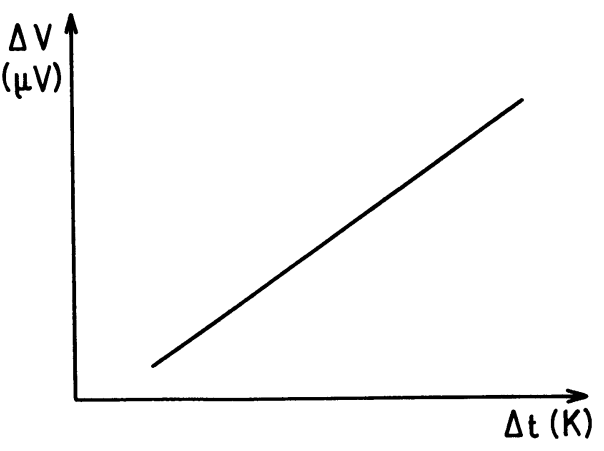

Fig. 6. - Enregistrement théorique $\Delta V=f(\Delta T)$.

[Theoretical plot of $\Delta V v s .(\Delta T)$.]

est possible de mesurer le pouvoir thermoélectrique à la température considérée. Pour qu'à très basse fréquence $\left(f \sim 5 \times 10^{-2} \mathrm{~Hz}\right)$, le gradient thermique suive la forme d'onde du courant de chauffe dans l'ampoule, on choisit pour ce dernier la forme :

$$
I=u_{\mathrm{t}} \cdot\left(I_{\mathrm{m}} \sin ^{2} \omega t\right)+I_{0}
$$

(où $u_{\mathrm{t}}$ représente la fonction échelon). Le courant constant $I_{0}$ maintient un léger rougeoiment du filament, ce qui supprime le seuil de tension en dessous duquel l'émission est négligeable.

Les tensions proportionnelles au gradient thermique $\Delta T$ et à la tension de Seebeck $\Delta V$, sont enregistrées simultanément sur les deux voies d'un enregistreur X-Y (Figs. 6 et 7).

Le graphe théorique ainsi obtenu est un segment de droite dont la pente est proportionnelle au coefficient de Seebeck. Si les contacts thermiques entre l'échantillon et les blocs chaud et froid sont suffisants, la courbe expérimentale s'éloigne peu de la droite théorique (Fig. 6). Des thermocouples trop éloignés de l'échantillon créent un retard entre l'excitation thermique et la tension résultante; si le thermocouple (k) reçoit le flux thermique avant l'échantillon, la courbe de réponse devient alors ellipsoïdale (Fig. 7). Un appariement insuffisant des filtres moyenneurs conduit au même résultat.

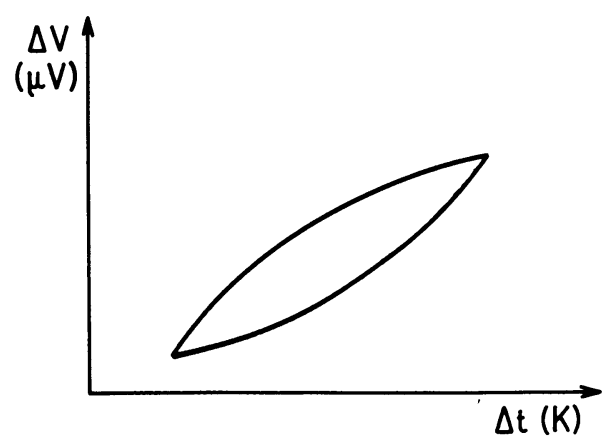

Fig. 7. $-\Delta V=f(\Delta T)$ mise en évidence d'un défaut d'appariement des voies de mesure.

[Evidence of a pairing defect between $\Delta T$ and $\Delta V$ channels.]
Lorsque la résistance $R$ de l'échantillon augmente, le temps d'établissement de la tension $\Delta V$ croît en fonction de la constante de temps $\tau_{1}=R C_{\mathrm{p}}$. La constante de temps de la voie qui enregistre le gradient thermique est égale au produit : $\tau_{2}=r C_{\mathrm{p}}(r$ : résistance du thermocouple, $C_{\mathrm{p}}$ capacité parasite de l'amplificateur $\left.\mathbf{A}_{4}\right)$. L'inégalité $\tau_{2} \ll \tau_{1}$ impose que les deux voies de mesures ne puissent être en phase que si la période de l'excitation est très inférieure à la constante $\tau_{1}$. Pour des résistances $R \simeq 10^{9} \Omega$, on utilise par exemple un signal de fréquence $F<10^{-2} \mathrm{~Hz}$.

4. Résultats des mesures. - Grâce à l'appareil ainsi construit, il est possible de mesurer des pouvoirs thermoélectriques de l'ordre de $20 \mu \mathrm{V} / \mathrm{K}$ sur des échantillons de surface de contact aussi faible que $0,5 \mathrm{~mm}^{2}$ et dont la résistance atteint $10^{10} \Omega$. Pour un même échantillon, monté de la même manière, l'écart entre deux manipulations successives n'excède pas $5 \%$.

La cellule a été contrôlée à l'aide d'un échantillon monocristallin de $\mathrm{Fe}_{3} \mathrm{O}_{4}$ stœchiométrique. L'échantillon sur lequel nous avons effectué les mesures avait été testé antérieurement par Simsa [5] avec un équipement tout à fait différent. Ce dernier mettait en jeu un cryostat à convection et un électromètre à capacité vibrante. La mesure du gradient thermique était réalisée à l'aide de deux thermocouples isolés électriquement de l'échantillon. Cette cellule ne pouvait fonctionner qu'avec des échantillons d'au moins $1 \mathrm{~cm}$ de long, condition qui n'est pas toujours réalisable lorsqu'on étudie des monocristaux.

Nous avons effectué la manipulation sur un fragment cubique (de $2 \mathrm{~mm}$ de côté) issu du même échan-

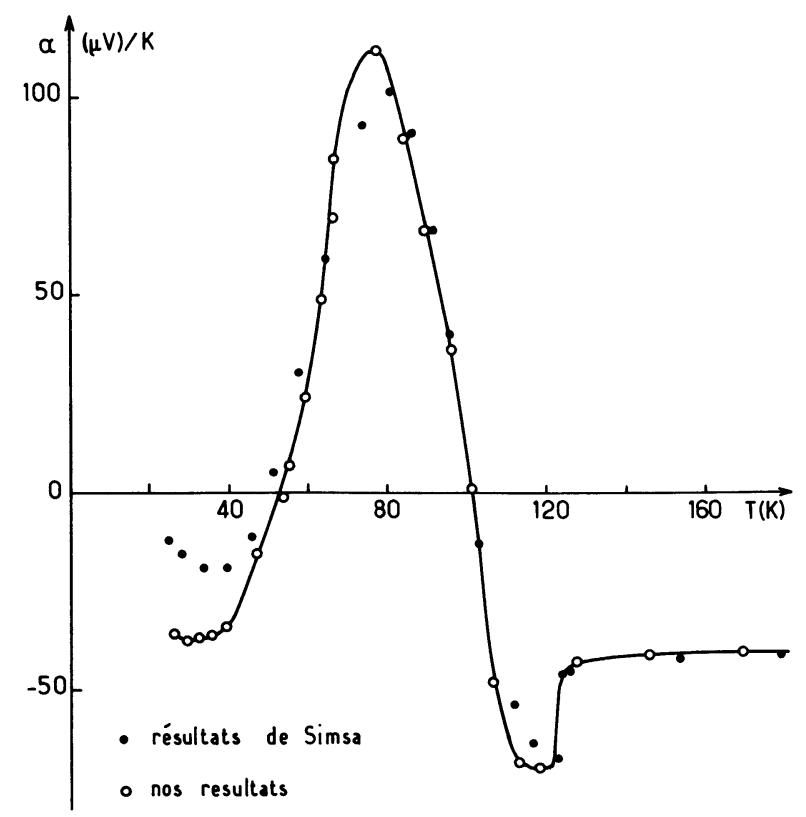

Fig. 8. - Pouvoir thermoélectrique de la magnétite stœchiométrique en fonction de la température.

[Thermoelectric power is. temperature in a stoichiometric sample of magnetite.] 
tillon. Le domaine de température exploré s'étendait de 20 à $300 \mathrm{~K}$. Dans cet intervalle de température, la résistivité de l'échantillon variait de $5 \times 10^{9} \Omega \mathrm{cm}$ à $10^{-2} \Omega \mathrm{cm}$.

Les courbes représentées à la figure 8 reproduisent les résultats obtenus par les deux méthodes. Les températures de transition de Verwey et de changement de signe du coefficient de Seebeck sont très voisines. A basse température, les écarts obtenus sur la valeur du pouvoir thermoélectrique entre les deux méthodes de mesures peuvent être attribués à une certaine hétérogénéité du taux de répartition de défauts (chimiques et structuraux) dans le cristal utilisé par Simsa. L'influence de celle-ci deviendrait sensible au-dessous de $50 \mathrm{~K}$. Ces résultats sont en assez bon accord qualitatif avec ceux obtenus par Kuipers et Brabers [6] sur des échantillons provenant toutefois de préparations différentes. Ces derniers auteurs n'ont pas exploré le domaine des températures inférieures à $80 \mathrm{~K}$.

L'appareil construit nous a permis en outre de déterminer le pouvoir thermoélectrique des hexaborures de terres rares $\mathrm{EuB}_{6}$ et $\mathrm{YbB}_{6}$ [7]. Il a également été utilisé pour la caractérisation des propriétés de transport des bronzes de tungstène substitués $\mathrm{Na}_{x} \mathrm{Ta}_{y} \mathrm{~W}_{1-y} \mathrm{O}_{3}$ au voisinage de la transition métalnon métal [8].

Remerciements. - Les auteurs souhaitent remercier $\mathbf{Z}$. Simsa et J. Keem pour les discussions fructueuses qui ont permis d'améliorer les performances de leur appareillage. L'un d'entre nous (P. D.) exprime sa reconnaissance au Professeur Honig et à ses collaborateurs pour leur hospitalité au cours du stage effectué à la Purdue University.

\section{Bibliographie}

[1] Mотт, N. F., Electronic processes in non-crystalline materials (Clarendon Press) 1971.

[2] Freeman, R. H. and Bass, J. B., Rev. Sci. Instrum. 41 (1970) 1171.

[3] KeEm, J., Communication privée.

[4] Balslew, I. and Hougs, E., J. Phys. E. 7 (1974) 811.

[5] Simsa, Z., Communication privée.
[6] Kuipers, A. J. M. and Brabers, V. A., Phys. Rev. B 14 (1976) 1401.

[7] Tarascon, J. M., Etourneau, J., Dordor, P., Hagenmuller, P., Kasaya, M., Coey, J. M. D., J. Appl. Phys. 51 (1) (1980).

[8] Doumerc, J. P., Dordor, P., Marquestaut, E., Pouchard, M., Hagenmuller, P., Philos. Mag., accepté pour publication. 